\title{
La coopération internationale sur les transferts de fonds des migrants, quels enjeux pour quelle perspective?
}

International Cooperation on Migrants' Remittances, Which Stakes for What

Perspective?

La cooperación internacional en torno al envío de remesas de emigrantes ¿Qué desafíos para qué perspectiva?

Marie Coiffard

\section{(2) OpenEdition}

Journals

Édition électronique

URL : https://journals.openedition.org/remi/5817

DOI : $10.4000 /$ remi. 5817

ISSN : $1777-5418$

Éditeur

Université de Poitiers

\section{Édition imprimée}

Date de publication : 1 mars 2012

Pagination : $129-145$

ISBN : 979-10-90426-03-0

ISSN : 0765-0752

Référence électronique

Marie Coiffard, «La coopération internationale sur les transferts de fonds des migrants, quels enjeux pour quelle perspective? », Revue européenne des migrations internationales [En ligne], vol. $28-\mathrm{n}^{\circ} 1$ । 2012, mis en ligne le 01 mars 2015, consulté le 16 avril 2022. URL : http://journals.openedition.org/ remi/5817 ; DOI : https://doi.org/10.4000/remi.5817 


\title{
La coopération internationale sur les transferts de fonds des migrants, quels enjeux pour quelle perspective?
}

\author{
Marie COIFFARD ${ }^{1}$
}

\section{INTRODUCTION}

T 'objet de cet article est la coopération internationale concernant des trans_ferts de fonds des migrants (TFM), phénomène transnational composé d'une double liaison, entre le migrant et son pays d'origine, et entre les pays envoyeurs et receveurs de TFM. L'objectif est ici d'appliquer une analyse d'économie politique internationale à cette seconde liaison, afin d'en tirer un enseignement quant à sa portée potentielle. D'après la base de données en ligne de la Commission des Nations Unies pour le Commerce et le Développement (CNUCED), les TFM vers les pays en développement (PED) ont augmenté de $270 \%$ entre 2000 et 2010. Après un ralentissement lié à la crise financière internationale en 2009, les TFM reprennent leur croissance en 2010 et dépassent 325 milliards de dollars. Les TFM sont ainsi devenus une source de financement extérieur plus importante que l'aide publique au développement (APD). Celle-ci a progressé deux fois moins vite que les TFM sur les dix dernières années (son montant global atteint 126 milliards de dollars en $2009^{2}$ ).

Cette nouvelle visibilité des TFM conduit de nombreux auteurs à les considérer comme une potentielle « manne » financière pour le développement (Kapur, 2004). Prenant appui sur ces travaux soulignant le potentiel « développementaliste » des TFM (Taylor, 1999), les organisations internationales telles que la Banque mondiale ou le Fonds monétaire international ont pris le parti d'inclure ces flux dans leur politique de développement. Dans ce cadre, une initiative globale est mise en place en 2004 appelée « Global Remittance Initiative » (GRI) par le biais du G8 (principaux pays d'envoi des TFM), visant à augmenter l'impact positif des TFM sur le développement des pays receveurs.

1 A.T.E.R. à la Faculté d'économie de Grenoble, Centre de recherche en économie de Grenoble (C.R.E.G.), BP 47, 38040 Grenoble cedex 9 ; marie.coiffard@upmf-grenoble.fr

2 Les données pour l'APD sont disponibles jusqu'en 2009 sur le site de la CNUCED. 
Cette coopération se révèle être au centre d'une double imbrication : de l'économique et du politique par la nature des flux et des acteurs concernés, et du national et de l'international. C'est pourquoi l'économie politique internationale apparaît comme une démarche pertinente pour analyser ce phénomène (Gilpin, 1987). Le concept de régime international qui qualifie certaines formes de coopération est ici mobilisé (et décrit plus avant dans la seconde partie de l'article). Dans ce contexte, cet article s'interroge sur les enjeux de la coopération internationale qui s'est développée au début des années 2000 sur les TFM, et propose de tirer quelques enseignements. Que nous apprend la théorie des régimes internationaux sur la portée de cette coopération?

La coopération internationale sur les TFM s'apparente à un régime de coordination (voir infra) au sens de Stein (1982). Par conséquent, la portée d'un tel régime est faible, celui-ci se révélant inadapté pour augmenter l'impact positif des TFM sur le développement économique des pays receveurs. La formation d'une coopération internationale sur les TFM fait suite à l'importante croissance des TFM depuis les années 1990, rendant inéluctable l'intégration de ces flux dans les préconisations des organisations internationales. Les enjeux de cette coopération sont donc finalement l'intégration des TFM dans un régime international plus large, portant sur le financement du développement.

Afin de mettre en évidence cette thèse, deux démarches sont mobilisées. Dans un premier temps, une analyse économique de l'évolution récente des TFM révèle que ces flux ont acquis une visibilité récente, au moment où l'aide publique au développement connaît une diminution importante. Les organisations internationales se sont alors emparées du sujet, soutenues par de nombreux travaux soulignant l'impact positif des TFM qui d'une part, participent à la diminution de la pauvreté et d'autre part, présentent un caractère contracyclique en cas de détérioration des conditions économiques du pays d'origine. Cette analyse montre les enjeux de la coopération qui sont à la fois un outil de développement potentiel pour les pays d'origine et un faible sacrifice économique pour les pays d'accueil. Dans un second temps, une analyse d'économie politique de la coopération internationale identifie la seule initiative d'envergure mondiale portant sur le domaine : la GRI. Les contours de cette coopération sont ainsi définis et confrontés à la théorie des régimes internationaux. La faible portée du régime apparaît alors évidente, alors que se dessine la possibilité d'un régime international plus large portant sur le financement du développement, dont le consensus de Monterrey pose les bases en 2002. Ce consensus résulte de la conférence des Nations Unies sur le financement du développement, qui fait émerger les sources de financement privées (telles que les TFM) comme les éléments désormais centraux du développement des PED. Cette piste est explorée en conclusion, afin d'insérer la coopération sur les TFM dans un contexte plus large. 


\section{LES TRANSFERTS DE FONDS DES MIGRANTS : UN «NOUVEAU» FLUX TRANSNATIONAL?}

Les TFM sont devenus en quelques années une composante importante des flux internationaux de capitaux et, pour de nombreux pays en développement, une des premières sources de devises et de financement de l'économie. Leur position dans la hiérarchie des sujets à enjeux dans les relations internationales s'est ainsi considérablement élevée - ce qu'atteste, entre autres, la floraison de rapports et d'études qui leur sont dédiés par les organismes publics nationaux et internationaux, ainsi que la multiplication de travaux académiques sur le sujet. Pour le vérifier, il est toutefois nécessaire de faire un retour sur la définition et les problèmes de mesure des TFM ainsi que sur leurs tendances récentes. Cette première analyse de l'économie des TFM permet de mettre en exergue les différents intérêts des pays d'accueil et d'origine des migrants.

\section{L'augmentation spectaculaire des TFM}

Les TFM sont constitués selon le Fonds monétaire international (FMI) par la somme des flux transitant par trois postes de la balance des paiements. Ils recouvrent les envois de fonds des travailleurs, la rémunération des salariés et les transferts des migrants. Les TFM sont donc des flux monétaires envoyés par un migrant dans un pays d'accueil vers un agent dans son pays d'origine ${ }^{3}$. Le FMI définit ces transferts comme : «le revenu des ménages issu d'économies étrangères, provenant principalement des mouvements de personnes, temporaires ou permanents, vers ces économies. [...] Ils consistent principalement en flux financiers et non-financiers envoyés ou donnés par des individus qui ont migré dans une nouvelle économie et y sont devenus résidents » (FMI, 2009 : 272).

Par définition, les transferts dits informels n'y sont pas comptabilisés, de même que les transferts en nature. Il existe trois types de transferts informels : le transfert direct via un déplacement physique du migrant ou d'un émissaire ; l'envoi par courrier ; et les transferts de type Hawala qui désigne, selon M. El Qorchi (2002 : 31), « un réseau informel de transfert de fonds d'un lieu à un autre par le biais de courtiers - les hawaladars -, quels que soient la nature de la transaction ou les pays impliqués. Si ce type d'opération est engagé le plus souvent par des travailleurs qui ont émigré dans un pays développé, il sert aussi à envoyer des fonds à partir d'un pays en développement, même si le but du transfert est en général différent $»$.

D’après Freund et Spatafora (2008), leur montant pourrait représenter entre $50 \%$ et $250 \%$ des transferts formels - ceux qui sont enregistrés dans les balances des paiements. Ce point statistique conditionne sérieusement l'étude des TFM et incite à la plus grande prudence quant à la portée des conclusions tirées des seuls flux enregistrés. Quelques enseignements associés à des évolutions marquantes des flux formels peuvent toutefois être tirés. Les TFM formels atteignent désormais des volumes comparables aux investissements directs étrangers (IDE) pour de nombreux PED (Graphique 1). En effet, les flux d'IDE à destination des PED dépassent 500 milliards de dollars en 2010 (CNUCED,

3 Par convention, les termes « pays d'accueil » et « pays d'envoi » se référeront toujours au pays d'accueil du migrant, et « pays d'origine » et « pays receveur » au pays d'origine du migrant. 
$2011^{4}$ ) et leur croissance sur les dix dernières années (entre 2000 et 2010) aura été de 122 \%. La croissance des TFM apparaît donc particulièrement dynamique.

Graphique 1 : Évolution des TFM, de l'APD et de l'IDE vers les pays en développement de 1980 à 2010

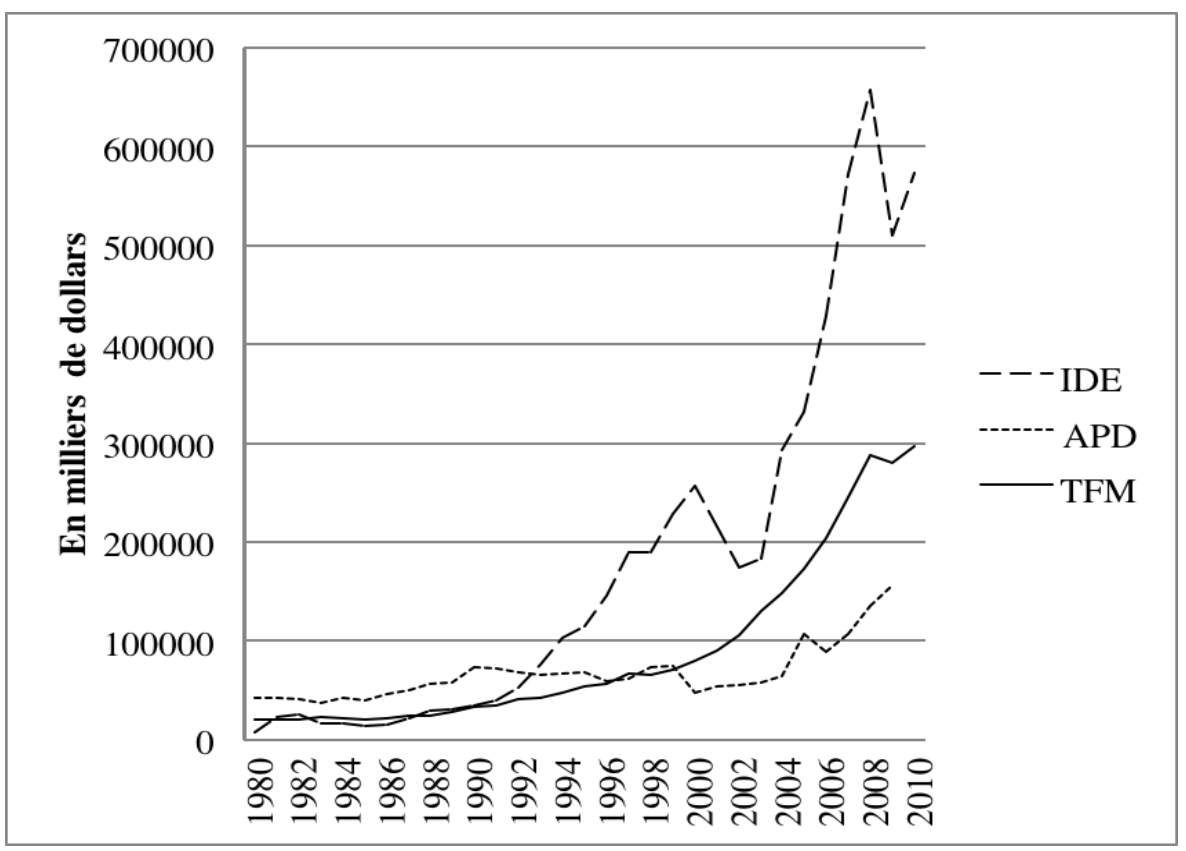

Source : CNUCED, 2011.

Cet essor peut être expliqué par différents facteurs, techniques et structurels. Tout d'abord, les éléments plutôt techniques affectent artificiellement la croissance des TFM. En effet, depuis les années 1970, l'amélioration des systèmes de comptabilité nationale dans les PED a permis d'enregistrer une plus grande partie des TFM. Ensuite, la globalisation financière, en multipliant les modes de transmission formels, a également favorisé l'essor des TFM depuis les années 1980-1990. Enfin, l'augmentation du nombre de travailleurs migrants peut expliquer cette croissance. L'une des conséquences de cette augmentation des TFM est la place prépondérante que prennent ces flux dans l'économie des pays qui les reçoivent. Le graphique 2 présente les vingt premiers pays receveurs de TFM en $\%$ du PIB. Pour chacun d'entre eux, les TFM équivalent à plus de $10 \%$ de leur production intérieure.

4 Ces données sont indicatives, car les investissements directs étrangers (IDE) enregistrent de fortes variations annuelles. 
Graphique 2 : Les vingt premiers receveurs de TFM en 2010 en \% de PIB

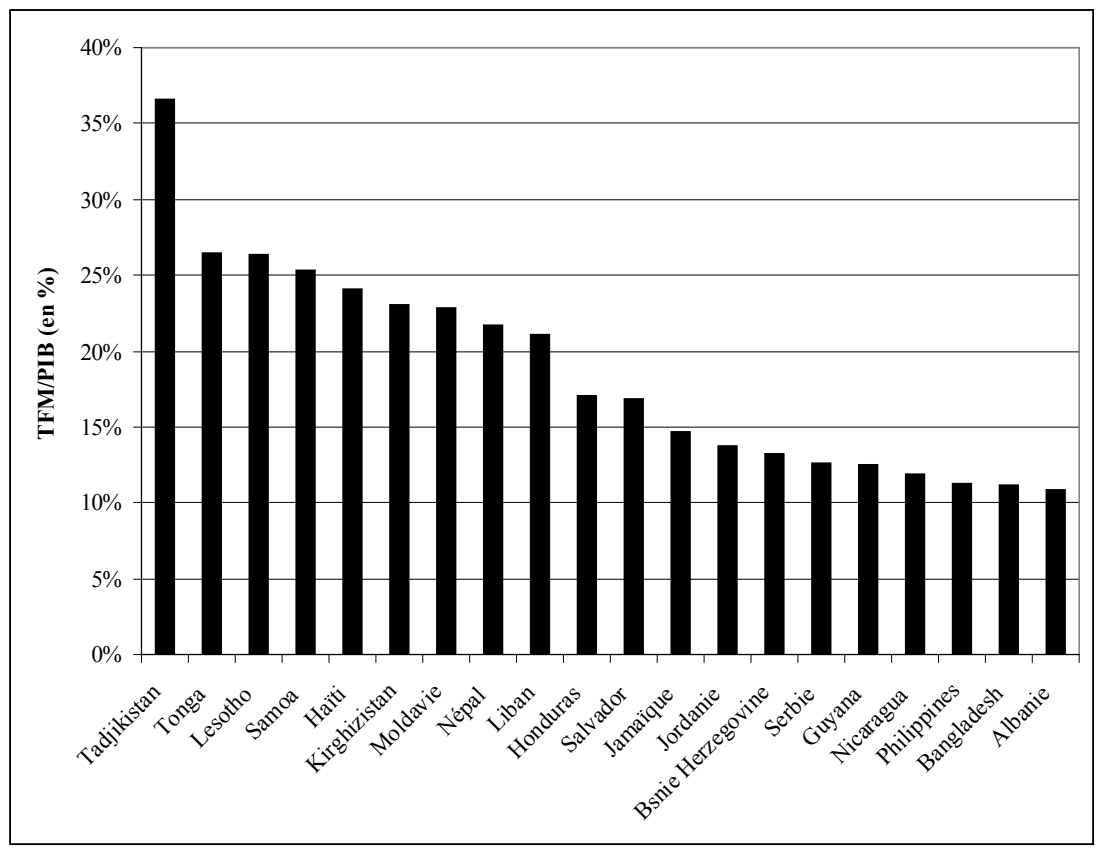

Source : CNUCED, 2011.

Cette évolution récente des TFM, en partie expliquée par un meilleur enregistrement de ces flux, semble avoir révélé un nouvel enjeu, dans les pays d'origine des migrants, mais aussi dans les pays d'accueil.

\section{L'impact des TFM sur les pays d'accueil et d'origine : un enjeu de développement}

La coopération sur les TFM relève de deux dimensions. La première, qui bien qu'assez technique n'en est pas moins un enjeu important, relève d'une logique de normalisation, d'adoption de standards internationaux. Mais la question des TFM ne se limite pas à une question technique. La seconde dimension relève de la résolution d'un problème d'action collective en présence d'intérêts hétérogènes. Afin de déterminer les enjeux d'une coopération internationale sur les TFM, il est nécessaire de définir les enjeux pour les participants potentiels. Ces flux entrants impactent directement l'économie des pays receveurs, alors qu'ils représentent des volumes monétaires relativement faibles pour les pays émetteurs.

L'impact des TFM sur les pays d'origine des migrants fait débat. Enregistrés au crédit de la balance des paiements, les TFM augmentent les ressources disponibles pour la plupart des PED. L'apport de devises bénéficie au revenu national, à l'épargne et à l'investissement ; en contribuant au financement des importations, il réduit les déséquilibres, voire les risques de crises de balance des paiements. Les TFM remplissent ainsi 
une fonction similaire aux flux de capitaux privés et publics (Russel, 1986). Cependant, certains auteurs soulignent que les TFM peuvent avoir un impact négatif comparable au « syndrome hollandais ${ }^{5}$ » (Reichert, 1981 ; Connell et Conway, 2000). Si l'augmentation de la demande est supérieure à l'augmentation des capacités de production, l'économie du pays récipiendaire connaîtra un biais dans ses importations en faveur des biens échangeables, avec en contrepartie un effet inflationniste sur le secteur non-échangeable. Il y a alors déplacement des ressources du secteur échangeable vers le secteur non échangeable, une détérioration de la position extérieure du pays et une diminution du bien-être des familles qui ne reçoivent pas de transferts (McCormick et Wahba, 2000 ; Reichert, 1981).

Les États qui souhaitent optimiser ces transferts vont alors chercher à mettre en place des régulations nationales. Dans la mesure où le marché des TFM n'est pas parfait, le coût de la non intervention de l'État correspond à un coût d'opportunité, si l'intervention étatique permettait de réduire les coûts de transaction. La régulation des TFM peut avoir un impact sur ces coûts et cette régulation résulte des politiques nationales mises en place par les États. Les pays d'origine ont un intérêt direct dans la mise en place d'une régulation permettant d'augmenter le volume et l'impact positif des TFM. Pour les pays d'accueil au contraire, les TFM représentent une perte économique. Pourtant, les pays du G8 ont lancé la Global Remittance Initiative (GRI) visant à augmenter le volume des TFM en 2004. Ces pays sont selon les données de la CNUCED les premiers pays émetteurs de transferts en direction des PED (cf. Tableau 1). Par conséquent, que ce soit pour permettre le financement des Objectifs du Millénaire pour le Développement (OMD), comme le suggère le consensus de Monterrey (2002) ou bien pour compenser une réduction de l'APD, les sommes envoyées depuis ces pays vers les PED semblent faibles, comparées au PIB national. Plus de 36 milliards de dollars ont quitté les États-Unis en 2003 (l'année précédant le lancement de l'initiative), ce qui correspond à $0,33 \%$ du PIB national. Depuis 2003, ces flux sortants ont augmenté pour atteindre près de 50 milliards de dollars en 2009.

Tableau 1 : Les dix principaux pays d'envoi des TFM

\begin{tabular}{|l|r|r|}
\hline \multicolumn{1}{|c|}{ Flux sortants } & $\mathbf{2 0 0 3}$ & $\mathbf{2 0 0 9}$ \\
\hline États-Unis & $36544^{*}(0.33)^{* *}$ & $47553(0.34)$ \\
\hline Arabie Saoudite & $14783(6.89)$ & $25969(7.14)$ \\
\hline Suisse & $11451(3.49)$ & $19174(3.90)$ \\
\hline Allemagne & $11189(0.46)$ & $15924(0.48)$ \\
\hline Espagne & $5140(0.58)$ & $12610(0.86)$ \\
\hline Luxembourg & $5010(17.18)$ & $10556(21.77)$ \\
\hline France & $4388(0.24)$ & $5224(0.20)$ \\
\hline Italie & $4368(0.29)$ & $12986(0.61)$ \\
\hline Pays-Bas & $4238(0.79)$ & $3670(1.02)$ \\
\hline Royaume-Uni & $2623(0.14)$ & $3.17)$ \\
\hline$*$ en millions de dollars, prix et taux de change courants ** en pourcentage du PIB \\
\hline
\end{tabular}

Source : CNUCED, 2011.

5 Selon Cottenet-Djoufelkit (2003:5) «le modèle de base de cette théorie explique comment un choc externe positif engendre la désindustrialisation d'une petite économie ouverte, comme ce fut le cas en Hollande après la découverte de pétrole sur son territoire». 
Les TFM sont un véritable enjeu de développement pour les pays qui les reçoivent, car ils représentent une part de plus en plus importante de leur PIB. Cependant, ces flux n'ont pas que des effets positifs comme le démontre l'existence de deux courants différents dans la « Nouvelle économie des migrations de travail ». Le premier est qualifié de « développementaliste » (Taylor, 1999) et accepte l'hypothèse d'un impact globalement positif des TFM. Au contraire, l'approche " pessimiste » refuse cette assertion en soulignant les effets négatifs entrainés sur l'économie.

En premier lieu, la décision migratoire est conçue comme une stratégie du ménage pour maximiser son revenu, obtenir des fonds pour investir dans de nouvelles activités et mettre en place un mécanisme d'assurance permettant de diversifier les risques liés au revenu de la famille et la production nationale. De nombreux travaux empiriques ont montré qu'il existe un lien fort entre les TFM et les chocs subis par les familles de migrants dans leur pays d'origine, tels que des chocs agricoles comme une sécheresse (Lucas et Stark, 1985 ; Azam et Gubert, 2005), ou encore des conflits armés (Lindley, 2009 sur la Somalie). Ainsi, un choc diminuant le bien-être de la famille du migrant est suivi d'une augmentation des TFM. Cette caractéristique contracyclique est également observée par Chami et al. (2005) sur un panel de 113 pays au niveau macroéconomique.

En second lieu, les TFM, voire les TFM potentiels (liés au salaire espéré), sont à l'origine d'une «dynamique de développement [mise en place] par le relâchement des contraintes de production et d'investissement auxquels font face les ménages dans les pays pauvres en développement» (Taylor, 1999 : 64). Cette «dynamique de développement» implique selon Taylor que les TFM aient un impact sur l'investissement et la production du pays d'origine. Cette hypothèse a été testée et validée par plusieurs études, par exemple celle de Woodruff (2007) sur l'impact des TFM sur la création et le financement de petites et moyennes entreprises à Mexico.

Un second courant d'analyse des TFM nourri par Reichert (1981) ou encore Wiest (1984) soutient l'idée que les TFM et les flux migratoires peuvent avoir un impact négatif sur les économies d'origine. Cet impact est renforcé par le caractère auto-entretenu des migrations, qui conduit les pays à se «spécialiser » dans l'export de migrants, provoquant une sorte de « syndrome hollandais ». Cette thèse est soutenue par quelques travaux, notamment ceux de Ruiz (2008) sur les Philippines. Cet économiste de la Banque mondiale montre comment l'État Philippin a structuré la société et notamment le système éducatif en fonction d'une politique de promotion de l'émigration. Selon certaines études, les TFM sont plus consommés qu'investis (Taylor et al., 1996 ; Durand et Massey, 1992 ; Papademetriou et Martin, 1991). Cependant ainsi que le reconnaissent Taylor et al. (2005), ces études empiriques reposent sur une définition restrictive de « l'investissement productif », qui n'inclut pas, par exemple, les dépenses d'éducation. Dans cette optique, les TFM peuvent donc avoir un impact négatif sur le développement des pays d'origine. Il est clair alors que l'intérêt des pays receveurs est de faciliter les TFM tout en en limitant les impacts négatifs. Pour les pays d'envoi, ces flux ont un faible poids économique. L'initiation d'une coopération internationale sur ces flux par les pays d'envoi repose donc sur d'autres intérêts. 
L'apparition de forums ou de processus consultatifs plus ou moins formels au niveau international et régional révèle l'émergence d'une demande de régulation au niveau global. Cette demande coïncide avec l'apparition d'un relatif consensus au niveau international sur l'intérêt d'une régulation globale des TFM visant à maximiser les TFM et leurs effets bénéfiques dans les pays d'origine. Ce consensus correspond à la reconnaissance d'une " issue area », socle indispensable à la mise en place d'un régime international (Haas, 1980). L'Organisation internationale des migrations (OIM), la Banque interaméricaine de développement, la Banque asiatique de développement, la Banque Africaine de développement ou encore les commissions régionales des Nations Unies, reconnaissent de plus en plus l'impact potentiellement positif des TFM sur les économies d'origine. «Les TFM peuvent promouvoir le développement si un environnement favorable pour l'utilisation des TFM, des arrangements institutionnels pour les transferts monétaires et la disponibilité d'opportunités d'investissements existent dans le pays d'origine » (OIM, 2009). Cette reconnaissance se retrouve également au travers des nombreux programmes mis en place par les agences de développement régionales qui reconnaissent toutes la baisse des coûts de transaction, la promotion des partenariats institutionnels, la bancarisation des migrants et la promotion des investissements productifs comme des éléments essentiels de l'établissement de politiques visant l'optimisation des TFM. Au sein des commissions régionales des Nations Unies, ces programmes s'intègrent également aux objectifs de financement des OMD. À l'échelle internationale depuis la fin des années 1990, émerge un relatif consensus sur l'impact potentiellement positif des TFM couplé à la mise en place de coopérations, partenariats et/ou de politiques nationales propices. Cependant, ces programmes recouvrent des périmètres nationaux, voire régionaux, et bien qu'ayant des objectifs similaires, ne font pas l'objet de règles ou procédures homogènes. Ce consensus est enfin formalisé au niveau global par le lancement de la GRI en 2004, impliquant particulièrement la Banque mondiale et la Banque des règlements internationaux.

\section{L'ÉCONOMIE POLITIQUE DES TFM : UN RÉGIME PEU AMBITIEUX QUI VISE À RENFORCER LA VISIBILITÉ DES TFM PAR DES PRATIQUES DE NORMALISATION}

L'implication certes croissante mais très variée des États dans la régulation des TFM ainsi que la multiplication des forums mondiaux et régionaux sur cette question soulignent le manque de régulation globale des TFM. De plus, la nature des acteurs impliqués et l'enjeu économique et politique que les TFM représentent conduisent à s'interroger sur les motivations des États à coopérer, et donc à rechercher des coûts liés à l'absence de coopération. Le cadre multidimensionnel et transdisciplinaire de la théorie des régimes internationaux permet d'une part d'identifier ces coûts et d'autre part, établit des critères empiriquement vérifiables.

\section{Le régime international comme un moyen de réduire les coûts de transaction}

Krasner (1983 : 2) définit le régime international comme un « ensemble [...] de principes, de normes, de règles et de procédures de prise de décision autour desquelles 
les anticipations des acteurs convergent dans un domaine donné des relations internationales ». La création de ce régime repose notamment sur l'importance de ces acteurs, et donc sur la participation des principales économies, ou de la principale économie. Son leadership est souvent à l'origine de la dynamique de construction du régime, ainsi que de son maintien. Selon Berthaud (2006), un régime international se définit comme «i) une procédure interétatique, ii) de normalisation ou de résolution d'un problème d'action collective, iii) dans un domaine donné des relations internationales et iv) son effectivité repose sur des règles à effet indirect qui laissent aux États participants des libertés (sinon toute liberté) pour en internaliser les effets, c'est-à-dire pour s'y adapter ». La notion de régime international permet ainsi d'analyser la coopération internationale sur un sujet donné, afin d'en appréhender la portée ou l'effectivité.

La création d'un régime international est un moyen pour un ensemble d'États de coopérer dans un domaine spécifique des relations internationales et ce, à deux niveaux possibles. Le premier niveau relève d'une logique de normalisation, d'adoption de standards internationaux, comme dans le cas du transport aérien ou de la normalisation comptable. Le second niveau relève de la résolution d'un problème d'action collective en présence d'intérêts hétérogènes, mais surtout conflictuels. "Dans cette perspective, un régime s'explique par l'intérêt rationnel des États à coopérer en vue de réduire les coûts de transaction, d'obtenir une meilleure information, de diminuer les incertitudes mutuelles et de rendre plus lourd le coût d'alternatives non désirées » (Graz, 2004 : 559). En effet selon Keohane (1982), la création d'un régime international permet notamment d'assurer une coopération internationale positive pour les participants, qui réduise les coûts de transactions engendrés par la coopération et qui augmente le coût de la noncoopération. La construction d'un régime international permet ainsi de diminuer les coûts de transaction auxquels font face les États participants.

Enfin, Stein (1982) différencie deux types de régimes internationaux, dont les modalités et les enjeux diffèrent. Les régimes peuvent prendre la forme d'une collaboration ou d'une coordination entre États. Les régimes émergent à partir de situations anarchiques ou conflictuelles qui engendrent des coûts, liés aux intérêts divergents des acteurs. Ces deux régimes n'ont pas la même envergure, car ils n'ont pas les mêmes objectifs. Le régime de collaboration correspond à la coopération qui permettra aux acteurs d'atteindre un équilibre supérieur à celui qui découle de l'absence de coopération. Les États ont ici un " intérêt commun » à coopérer. Dans le cas du régime de coordination, les États ne manifestent pas d'intérêts communs, mais une " aversion commune », c'est-à-dire une issue défavorable à tous les participants. Ces derniers ont intérêt à coopérer afin d'éviter cette issue, malgré des préférences parfois divergentes. Les intérêts des États différencient la nature du régime. Ces intérêts sont liés à la présence de coûts spécifiques, qu'il convient d'observer dans le cas des TFM.

\section{Les coûts liés à l'absence de régulation internationale, à l'origine de la formation d'un régime international}

Selon la définition de Berthaud (2006), la coopération sur la régulation des TFM peut effectivement faire l'objet d'un régime international. La régulation fait inter- 
venir les États comme acteurs principaux fixant les règles, les procédures, etc., que l'on retrouve dans la définition de Krasner (1983). Cependant, le rôle des acteurs privés dans cette régulation, et plus largement la place du « marché des transferts de fonds », nous conduit à nuancer cette position. Les transferts formels passent par de nombreux canaux (Tableau 2). Les deux modes de transmission principalement utilisés sont les sociétés de transferts d'argent (STA), Western Union par exemple, et le réseau bancaire. Les parts de marché de ces prestataires diffèrent sensiblement selon le système migratoire considéré. Les parts de marché mondiales sont mal connues. Selon Orozco (2002), Western Union, la plus importante des sociétés de transferts d'argent détiendrait en 2002 environ $26 \%$ des parts de marché mondiales (marché formel). Concernant l'Amérique latine et les Caraïbes, Les STA détiendraient environ $70 \%$ du marché, les banques approximativement $11 \%$.

Tableau 2 : Les différents canaux de transmission des TFM formels

\begin{tabular}{|l|l|l|l|}
\hline \multicolumn{1}{|c|}{$\begin{array}{c}\text { Prestataires de } \\
\text { services de transfert }\end{array}$} & \multicolumn{1}{|c|}{$\begin{array}{c}\text { Mécanismes de } \\
\text { transfert }\end{array}$} & \multicolumn{2}{c|}{ Méthodes de remise des fonds } \\
\hline Banque & $\begin{array}{l}\text { Chèques/traites de } \\
\text { banque }\end{array}$ & Détail/Guichet & $\begin{array}{l}\text { Kiosques } \\
\text { informatiques }\end{array}$ \\
\hline $\begin{array}{l}\text { Coopérative de crédit et } \\
\text { d'épargne }\end{array}$ & $\begin{array}{l}\text { CCA/SWIFT (réseau } \\
\text { interbancaire) }\end{array}$ & Guichets automatiques \\
\hline Banque postale & $\begin{array}{l}\text { Mandats/chèques } \\
\text { postaux }\end{array}$ & Téléphones fixes et mobiles \\
\hline $\begin{array}{l}\text { Société de transferts } \\
\text { d'argent }\end{array}$ & Réseaux spécialisés & Internet \\
\hline $\begin{array}{l}\text { Prestataire non } \\
\text { bancaire de services de } \\
\text { microfinance }\end{array}$ & & \\
\hline
\end{tabular}

Source : Isern, Deshpande et Van Doorn, 2005.

Le coût des TFM dépend du nombre et de la nature des opérateurs. Quel que soit le mode de transfert, le prix peut être découpé en trois parties. Le premier composant est constitué des frais à la charge de l'envoyeur, le second la commission de change et le dernier (facultatif) sont les frais à la charge du receveur. La commission de change correspond à la différence entre le taux de change du marché et le taux effectivement utilisé par le prestataire. De plus, les STA proposent plusieurs services de transferts. Par exemple chez MoneyGram les migrants peuvent utiliser le "same day service » qui coutera 18 \$ pour un envoi de $300 \$$ depuis New York au Bangladesh. Mais le migrant peut également utiliser le «Economy service » qui lui coutera 10 \$ en frais. Cependant, le montant perçu par le destinataire n'est qu'une estimation. Dans la mesure où le taux de change n'est pas fixe, MoneyGram se laisse le droit de modifier les frais selon le moment où le récipiendaire retirera l'envoi ${ }^{6}$.

6 Site de MoneyGram, consulté le 12 octobre 2007. URL : www.moneygram.com. 
Pour conclure, le coût des TFM dépend de la nature et du nombre d'opérateurs engagés dans la transaction, ainsi que des différentes commissions prélevées. Ce mécanisme a deux conséquences principales. La première pour les migrants eux-mêmes. Les migrants subissent un coût important lié à la recherche d'informations pour trouver l'opérateur le moins cher. Or cette information est souvent peu disponible. De nombreuses études montrent que le prix d'un transfert peut varier considérablement selon les opérateurs, mais également selon les villes d'envoi et d'arrivée du transfert, ainsi que le moment de la journée ou le migrant souhaite réaliser l'opération (Orozco, 2006). Le principal coût pour les migrants est celui de la recherche d'information. Le marché de TFM est peu transparent (DFID, 2005). Nous sommes dans un cas d'asymétrie d'information en faveur des prestataires de services financiers. Une étude d'Appleseed (2005) sur le corridor ÉtatsUnis-Mexique souligne le manque de transparence sur le marché des TFM, qui rend la comparaison des prix quasi impossible entre plusieurs compagnies : "en Géorgie, un consommateur peut avoir dépensé entre $\$ 3.88$ et $\$ 21.90$ pour le même jour en juin, pour envoyer $\$ 300$ à Mexico ». De plus, les prestataires de services sont souvent réticents à donner des détails sur les commissions qu'elles prélèvent. Selon Orozco $(2002 ; 2006)$, ce manque d'information pourrait être combattu par les gouvernements par la mise en place de régulation.

\section{La Global Remittance Initiative : un régime international de normalisation des pratiques}

En 2004, la réunion du G8 de Sea Island s'est soldée par le lancement d'une initiative d'envergure internationale, la GRI au sein d'un plan d'action international. Le premier thème de ce plan, intitulé « Faciliter les TFM pour aider les familles et les petites entreprises » reconnaît que les TFM peuvent «jouer un rôle central dans le développement du secteur privé, permettant aux familles de recevoir du capital pour, par exemple, l'éducation, le logement et le lancement et le développement de petites entreprises ». Pour parvenir à cet objectif, le G8 établit une liste de six objectifs :

i) Encourager le développement de réseaux de transferts formels, en améliorant « l'alphabétisation » financière des populations et en travaillant avec le secteur privé à l'élargissement des services proposés (par exemple, le développement des transferts par téléphones mobiles, en pleine progression notamment sur le continent américain) ;

ii) La réduction des coûts de transaction par l'intensification de la concurrence et l'utilisation de nouveaux instruments financiers (à l'image de l'évolution du marché nord américain);

iii) Une plus grande cohérence et une coordination renforcée entre les Organisations internationales pour l'amélioration des services de transferts et l'augmentation de leur impact sur le développement ;

iv) Une coopération renforcée entre les prestataires de services financiers aux migrants et les institutions financières locales (par exemple le développement de partenariats entre prestataires de services financiers et microfinance);

v) Encourager la création de fonds locaux de développement et d'union de crédit qui offrent aux récipiendaires des possibilités d'investissements productifs supplémentaires (exemple Égyptien) ; 
vi) La mise en place d'un dialogue entre gouvernement, société civile et secteur privé, qui permette le renforcement ou la création d'infrastructures spécifiques ainsi que la levée des obstacles réglementaires (par exemple, les gouvernements devraient assurer un accès non-discriminatoire aux systèmes de paiements pour le secteur privé, compatibles avec les standards bancaires internationaux et la modernisation des infrastructures financières nationales).

À la suite de cette réunion, un groupe de travail réunissant principalement la Banque mondiale et la Banque des règlements internationaux (BRI) est mis en place afin d'élaborer des «principes généraux sur la régulation des TFM». En 2006, le groupe publie ces principes généraux («General principles for International Remittance Services »). Pour chacun de ces principes, le rapport propose un ensemble de mesures qui peuvent être appliquées par les pays participants. Ces mesures constituent des règles à effet indirect devant être internalisées librement par les acteurs (Kébabdjian, 2004). L'approche retenue est clairement une approche de type anglo-saxonne, qui se focalise sur des objectifs d'efficacité de marché (augmentation de la concurrence, baisse des coûts, amélioration de la transparence et de l'information).

L'émergence de cette initiative globale correspond à la reconnaissance d'un domaine particulier de coopération ( issue area »), nécessaire à la construction d'un régime international (Haas, 1980). Ce domaine est distinct de la coopération sur les mouvements de travailleurs eux-mêmes. Cette dernière se limite finalement à un ensemble d'accords migratoires bilatéraux, ainsi qu'à une négociation réduite à l'OMC dans le cadre de l'Accord général sur le commerce des services. Autrement dit, il n'existe pas aujourd'hui de véritable coopération proprement internationale sur les migrations, malgré les appels de nombreuses personnalités, comme celle de l'économiste Bhagwati (2003), pour une gestion concertée de ces flux. Les TFM sont donc un domaine indépendant et spécialisé de négociation, et pas une externalité des flux humains.

Par ailleurs, la GRI est bien une procédure interétatique, qui a vocation à intégrer tous les États confrontés aux TFM. Les pays d'envoi en premier lieu, initiateurs de la coopération, mais également les pays receveurs qui en seraient les principaux bénéficiaires. Cette négociation se déroule dans différentes « arènes » dont les institutions internationales majeures (Banque mondiale notamment).

L'objectif est la normalisation des systèmes de transfert et dans une moindre mesure la résolution d'un problème d'action collective (amélioration de l'impact des transferts). Si les déclarations présentent l'initiative comme un moyen d'optimiser l'impact des TFM sur les pays receveurs, elle est constituée dans les faits par un ensemble de propositions visant à simplifier et à standardiser les pratiques des États. Sous couvert d'un objectif développementaliste, la GRI se révèle correspondre plutôt à un régime de coordination au sens de Stein (1982). Selon Stein, chaque État est intéressé par la maximisation de ses gains absolus et pas relatifs. C'est-à-dire que l'objectif de la négociation ou de la coopération ne peut être d'être " plus » gagnant que les autres parties. En conséquence, chaque État doit trouver un intérêt suffisant pour coopérer. L'étude de l'économie des TFM révèle que les États participants n'ont pas « d'intérêt commun » à proprement parler. L'analyse de Stein permet de dépasser ce problème en proposant comme autre objet de 
coopération : l'« aversion commune ». L'aversion commune dans le cas des TFM semble être l'absence de régulation, qui apparaît comme non souhaitable pour les pays d'envoi comme de réception. Selon Stein l'aversion commune ne nécessite pas de collaboration, mais requiert un certain degré de coordination. Tout comme dans la GRI, aucune sanction n'est prévue pour les participants qui feraient défection, le régime étant considéré comme « auto-disciplinant » (self-enforcing).

Enfin, le régime sur les TFM repose effectivement sur des règles à effets indirects telles que définies par Kébabdjian (1999). Les principes généraux se déclinent en cinq grands thèmes portant sur : i) la transparence et la protection des consommateurs ; ii) les infrastructures de paiement ; iii) l'environnement légal et réglementaire ; iv) la structure de marché et la concurrence ; v) la gestion de la gouvernance et du risque.

Pour chacun de ces domaines d'action, la GRI propose une série de mesures politiques qui sont susceptibles de permettre d'atteindre ces objectifs. Mais ces mesures ne sont que des propositions, qui n'ont pas valeurs d'obligation. Elles correspondent donc à des règles librement internalisables par les États comme l'indique Berthaud (2006).

En conclusion, la GRI correspond à un régime de coordination au sens de Stein. La GRI remplit les quatre grands critères définissant un régime international dans son sens général. Cependant, l'envergure de ce régime est limitée, car il correspond plus à une normalisation des pratiques liées à une aversion commune pour l'absence de règles qu'à la résolution d'un problème d'action collective. Le régime repose sur des règles ouvertes, et n'expose pas les participants à une quelconque sanction en cas de défection. La portée de ce régime apparaît comme particulièrement limitée. Cette faible envergure s'explique en réalité par l'insertion des TFM dans une nouvelle politique de financement du développement, amorcée en 2002 lors du Consensus de Monterrey réunissant les Nations Unies.

\section{CONCLUSION : UN NOUVEAU PARADIGME SUR LE FINANCE- MENT DU DÉVELOPPEMENT}

L'émergence de la question des TFM sur la scène internationale doit être reliée à deux autres évolutions - de nature très différente au demeurant. D'une part le recul tendanciel de l'aide publique au développement (APD) depuis la fin du XXe siècle qui donne d'autant plus d'importance aux TFM sur les sujets du financement du développement et de la négociation multilatérale. D'autre part, la formation d'un nouveau consensus sur le développement à l'issue de la conférence des Nations Unies de Monterrey en 2002.

Concernant l'APD, elle passe (en valeur courante) de 61 milliards USD en 1992 à 53 milliards USD en 2000, avant de se redresser et d'atteindre environ 72 milliards USD en 2007. Cette augmentation de $26 \%$ en sept ans est sans commune mesure avec celle des TFM : $214 \%$ sur la même période. Une telle évolution est révélatrice d'un changement profond et somme toute rapide des sources de financement du développement. Elle élève les TFM au rang de priorité dans l'agenda des négociations de la communauté internationale. 
D'autre part, l'adoption du consensus de Monterrey par les Nations Unies en 2002 est révélatrice d'une modification importante des priorités des pays développés en matière de financement du développement. Le « consensus de Monterrey » est le nom donné à l'accord auquel sont parvenus les États membres des Nations Unies au terme de la Conférence sur le Financement du Développement de 2002. Il s'agit d'une déclaration portée par les États-Unis et soutenue par les autres pays industrialisés. Les PED (le G77) s'exprimant par la voix de Chavez, ont tenu à marquer leur désaccord vis-à-vis de cette déclaration, sans pour autant se retirer du consensus. Seul Cuba s'y est totalement opposé.

La déclaration porte sur la mobilisation de l'épargne et elle pointe en particulier sur la promotion des sources privées de financement comme instrument pour atteindre les OMD : «Les pays étant de plus en plus dépendants les uns des autres sur le plan économique, il y a lieu d'adopter une conception globale des problèmes nationaux, internationaux et systémiques interdépendants que pose le financement du développement [...]. Notre action commune en faveur de la croissance, de l'élimination de la pauvreté et du développement durable nous impose une mission essentielle : instaurer dans chaque pays les conditions requises pour mobiliser l'épargne publique et privée qui servira à financer l'investissement nécessaire dans les biens productifs et le développement humain [...]. Un cadre national favorable est indispensable pour mobiliser les ressources nationales [...]. La communauté internationale devrait appuyer les efforts visant à la création d'un tel cadre ».

La réduction du coût des TFM (et donc potentiellement une augmentation de leur volume) entre dans la mobilisation des ressources intérieures : "Il importe également de réduire le coût des envois de fonds des travailleurs migrants dans leur pays et d'étudier les moyens d'encourager le placement de ces avoirs dans des activités de développement, $y$ compris le logement ». La question des TFM devient donc un sujet d'ordre mondial, qui concerne tant les PED que les pays industrialisés. Et si la réduction du coût des TFM s'inscrit au chapitre de la mobilisation des ressources nationales, le consensus indique que la mise en place d'un cadre favorable à cette mobilisation doit être soutenue par une action collective. Cette nécessité de l'action collective sur les TFM apparaît d'autant plus forte que la coopération multilatérale reste au point mort sur les migrations internationales de travail. Ainsi, la faible portée de la GRI s'explique par l'objectif même de la coopération : une aversion commune pour l'absence de règles, malgré des enjeux significatifs, démontre les limites des États sinon à « contrôler » du moins à encadrer des flux individuels transnationaux. 


\section{Références bibliographiques}

APPLESEED (2005) Creating a Fair Playing Field for Consumers: The Need for Transparency in the U.S.-Mexico Remittance Market, Washington DC, Appleseed, 14 p.

AZAM Jean-Paul and GUBERT Flore (2005) Those in Kayes. The Impact of Remittances on Their Recipients in Africa, Revue économique, 56 (6), pp. 1331-1358.

BERTHAUD Pierre (2006) Paradigmes et problématiques, in Pierre Berthaud et Gérard Kébabdjian Éds., La question politique en économie internationale, Paris, La Découverte, pp. 25-30.

BHAGWATI Jagdish (2003) The World Needs a New Body to Monitor Migration, Financial Times, October 24.

CHAMI Ralph, FULLENKAMP Connel and JAHJAH Samir (2005) Are Immigrant Remittance Flows a Source of Capital for Development?, IMF Staff Papers, 52 (1).

CNUCED (2011) Base de données en ligne.

CONNELL John and CONWAY Dennis (2000) Migration and remittances in island micro-states: a comparative perspective on the South Pacific and the Caribbean, International Journal of Urban and Regional Research, 24, pp. 52-78.

DEPARTMENNT FOR INTERNATIONAL DEVELOPMENT (2005) UK Remittance Market, DFID and UK Remittances Working Group Report.

DURAND Jorge and MASSEY Douglas S. (1992) Mexican Migration to the United States: A Critical Review, Latin American Research Review, 27, pp. 3-43.

EL-QORCHI Mohamed (2002) Hawala, Finance \& Development, 39 (4).

FONDS MONÉTAIRE INTERNATIONAL (2009) International Transactions in Remittances. Guide for Compilers and Users, Washington, IMF Editions, $94 \mathrm{p}$.

FREUND Caroline and SPATAFORA Nikolas (2008) Remittances: Transaction Costs, Determinants, and Informality, Journal of Development Economics, 86 (2), pp. 356-366.

G8 (2004) Applying the Power of Entrepreneurship to the Eradication of Poverty, G8 Action Plan, Sea Island, June 9.

HAAS Ernst B. (1980) Why Collaborate?: Issue-Linkage and International Regimes, World Politics, 32 (3), pp. 357-405.

GILPIN Robert (1987) The political economy of international relations, Princeton University Press, $472 \mathrm{p}$.

GRAZ Jean-Christophe (2004) La gouvernance de la mondialisation, Paris, La Découverte, 127 p.

ISERN Jennifer, DESHPANDE Rani and VAN DOORN Judith (2005) Crafting a Money Transfers Strategy: Guidance for Pro-Poor Financial Service Providers, CGAP Occasional Paper, 28 p.

KAPUR Devesh (2004) Remittances, the New Development Mantra?, G24 Discussion Paper Series, 29, United Nations Conference on Trade and Development.

KEBABDJIAN Gérard (2004) Économie politique du régionalisme : le cas euroméditerranéen, Région et développement, 1 (19), pp. 151-184.

KEBABDJIAN Gérard (1999) Les théories de l'économie politique internationale, Éditions du Seuil, Paris, 308 p.

KEOHANE Robert (1982) The Demande for International Regimes, International Organization, 36 (2), pp. 325-355.

KRASNER Stephen D. (Ed.) (1983) International Regimes, Cornell University Press, Ithaca, 384 p.

LINDLEY Anna (2009) Remittances in conflict: Some Conceptual Considerations, Journal of Economics and Statistics [Jahrbücher f. Nationalökonomie u. Statistik], 229 (6), pp. 774-786.

LUCAS Robert and STARK Oded (1985) Motivations to Remit: Evidence from Botswana, The Journal of Political Economy, 93, pp. 901-918.

McCORMICK Barry and WAHBA Jackline (2000) Overseas Employment and Remittances to a Dual Economy, The Economic Journal, 110 (463), pp. 509-534.

ORGANISATION INTERNATIONALE POUR LES MIGRATIONS (2009) Remittances, Developing Migration Policies, IOM website. 
ORGANISATION DES NATIONS UNIES (2002) Monterrey Consensus on Financing for Development, International Conference, Mars.

OROZCO Manuel (2006) International Flows of Remittances: Cost, competition and financial access in Latin America and the Caribbean toward an industry scorecard, Washington, DC, Inter-American Dialogue.

OROZCO Manuel (2002) Attracting Remittances: Market, Money and Reduced Costs, Paper Commissioned by the Multilateral Investment Fund of the Inter-American Development Bank.

PAPADEMETRIOU Demetrios G. and MARTIN Philip L. (Eds.) (1991) The Unsettled Relationship: Labour Migration and Economic Development, Westport, CN, Greenwood Press.

REICHERT Josh (1981) The Migrant Syndrome: Seasonal US Wage Labor and Rural Development in Central Mexico, Human Organization, 40 (1), pp. 56-66.

RUIZ Neil (2008) Managing Migration: Lessons from the Philippines, Migration and Development Brief, 6, The World Bank.

RUSSELL Sharan Stanton (1986) Remittances from International Migration: A Review in Perspectives, World Development, 14 (6), pp. 677-696.

STEIN Arthur A. (1982) Coordination and Collaboration: Regimes in an Anarchic World, International Organization, 36 (2), pp. 299-324.

TAYLOR J. Edward, MORA Jorge, ADAMS Richard H. and LOPEZ-FELDMAN Alejandro (2005) Remittances, Inequality and Poverty: Evidence from Rural Mexico, University of California, Davis, Department of Agricultural and Resource Economics.

TAYLOR J. Edward (1999) The New Economics of Labor Migration and the Role of Remittances in the Migration Process, International Migration, 37 (1), pp. 63-88.

TAYLOR J. Edward (1996) Migration internationale et développement économique : analyse au niveau d'une micro-économie, in J. Edward Taylor Éd., Stratégies du développement, de l'emploi et des migrations approches par la modélisation, Paris, OCDE, pp. 95-115.

WIEST Raymond E. (1984) External Dependency and the Perpetuation of Temporary Migration to the United States, in Richard C. Jones Ed., Patterns of Undocumented Migration: Mexico and the United States, NJ, Rowman and Allanheld, pp. 110-135.

WOODRUFF Christopher (2007) Mexican Microenterprise Investment and Employment: The Role of Remittances, Intal - ITD Working Paper, 26, Inter-American Development Bank. 


\section{La coopération internationale sur les transferts de fonds des migrants, quels enjeux pour quelle perspective?

\author{
Marie Coiffard
}

Cet article propose d'analyser les premiers pas de la coopération internationale portant sur les transferts de fonds des travailleurs migrants (TFM) au moyen des outils de l'économie politique internationale. En quelques années, les TFM sont devenus l'une des premières sources de devises pour les pays en développement. En plus d'être devenus un enjeu national, les TFM prennent une dimension internationale croissante en éveillant l'intérêt des institutions multilatérales et régionales. La question est alors de savoir si cette coopération, accompagnée de la formation d'un consensus international sur l'efficacité des TFM constitue une avancée vers un régime international. La détermination des objectifs de cette coopération, ainsi que des coûts qui s'y rattachent, nous conduisent à conclure que la GRI n'a que la faible envergure d'un régime de coordination au sens de Stein (1982). Cette initiative s'intègre en revanche dans une modification du consensus international sur le financement du développement.

\section{International Cooperation on Migrants' Remittances, Which Stakes for What Perspective?}

\section{Marie Coiffard}

This article focuses on the first steps of an international cooperation on migrants' remittances, using the tools of the international political economy. In recent years, remittances have become one of the main sources of foreign exchange for developing countries. In addition to becoming a national issue, remittances are a growing international dimension by awakening the interest of multilateral and regional institutions. The question then is whether this cooperation, backed by the formation of an international consensus on the effectiveness of remittances, is a step towards an international regime. Determining the objectives of this cooperation, as well as associated costs, lead us to conclude that the Global Remittance Initiative is an international coordination regime (Stein, 1982). Actually, this initiative is part of a recent evolution in the international consensus on financing for development.

\section{La cooperación internacional en torno al envío de remesas de emigrantes ¿Qué desafíos para qué perspectiva? \\ Marie Coiffard}

Este artículo propone un análisis de los primeros pasos de la cooperación internacional basada en las remesas de emigrantes (TFM según el acrónimo francés) con los instrumentos de la economía política internacional. En pocos años, las TFM han pasado a ser una de las primeras fuentes de divisas para los países en desarrollo. Además de haberse convertido en un tema de importancia nacional, las TFM toman una dimensión internacional creciente despertando el interés de las instituciones multilaterales y regionales. El asunto es entonces saber si esta cooperación, acompañada del surgimiento de un consenso internacional acerca de la eficacia de las TFM, significa un paso adelante hacia un régimen internacional. La fijación de los objetivos de esta cooperación, así como los costes inherentes a la misma, nos lleva a concluir que la «Global Remittance Initiative» apenas cuenta con una débil envergadura de un régimen de coordinación en el sentido de Stein (1982). Esta iniciativa se integra sin embargo en una transformación del consenso internacional sobre la financiación del desarrollo. 